\title{
Nonaccelerator study of the residual nuclear strong interaction
}

V.G. Plekhanov

Professor, Head of Fonoriton Sci. Lab., Garon Ltd, Lasnamae 22 - 3, Tallinn, 11413, Estonia

\author{
*Correspondence author \\ V.G. Plekhanov \\ Professor \\ Head of Fonoriton Sci. Lab. \\ Garon Ltd. \\ Lasnamae $22-3$ \\ Tallinn, 11413 \\ Estonia \\ E - mail : vgplekhanov@gmail.com
}

Submitted : 23 March 2020 ; Published : 28 April 2020

\begin{abstract}
The present status and recent developments in the nonaccelerator study of residual strong nuclear interaction in solids are briefly reviewed. The emphasis is on new experimental results and they interpretation which were not available in earlier reviews. Artificial activation of the strong interaction by adding of one neutron to the nucleus causes the global reconstruction of the macroscopic characteristics of solids. The experimental evidence of macroscopic manifestation of the strong interaction in optical spectra of solids which is different by term of one neutron from each other (using LiD crystals instead of $\mathrm{LiH}$ ) has been presented. This evidence is directly seen from luminescence (reflection) and scattering spectra. As far a the gravitation, electromagnetic and weak interactions are the same in both of kind crystals, it only emerges the strong interaction in deuterium nucleus. Therefore a sole conclusion is made that the renormalization of the energy of electromagnetic excitations (electrons, excitons, phonons) is carried out by the strong nuclear interaction. There is a common place in Standard Model of modern physics that the strong nuclear force does not act on leptons. Our experimental results show the violation of this strong conclusion. Moreover, observation of the isotopic shift phononless line in the photoluminescence (reflection) spectra of the whole series of $\mathrm{LiH}_{x} \mathrm{D}_{1-x}$ mixed crystals is permit to construct change of the strong interaction coupling, $\alpha \_\{s\}$, in the wide value range. The necessity to take into account the more close relation between quantum chromodynamics and quantum electrodynamics is underlined. In the first step the quantum electrodynamics should be taken into account the strong interaction at the description of elementary excitations (electrons, excitons, phonons) dynamics in solids. Our experimental results open a new avenue in the investigation of the hadron - lepton interaction via study the low - temperature characteristics (reflection, photoluminescence) of solids and may shed light on a number of fundamental puzzles in modern physics, particularly on the unification of forces.
\end{abstract}

\section{Keywords: Strong Interaction, Quarks, Gluons, Excitons, Phonons, Quantum Chromodynamics and Electrodynamics}

\section{Introduction}

Modern physics distinguishes three fundamental properties of atomic nuclei: mass, spin (and related magnetic moment), volume (surrounding field strength), which are the source of isotope effect. The stable elementary particles (electrons, protons and neutrons) have intrinsic properties. Some of these properties such as mass and electrical charge are the same for macroscopic objects. Some are purely quantum mechanical and have no macroscopic analog. Spin is an intrinsic angular momentum associated with elementary particles. The spin angular moment of an electron, measure along any particular direction, can only take on the values $\hbar / 2$ or $-\hbar / 2$. The nuclear magnetic moment associates with nuclear spin and produces the magnetic interaction with its environment. The ability

to manipulate and control electron and/or nucleus spin in semiconductor devices provides a new route to expand the capabilities of inorganic semiconductor - based electronics and to design innovative devices with potential application in quantum computing. One of the major challenges towards these objectives is to develop semiconductor - based systems and architectures in which the spatial distribution of spins and their properties can be controlled. For instance, to eliminate electron spin decoherence resulting from hyperfine interaction due to nuclear spin background, isotopically controlled nuclear spin - depleted devices are needed. The control of the spatial distribution of isotope with nuclear spins is a prerequisite 
to implement the quantum bits or qubits. Therefore stable semiconductor isotopes are important elements in the development of solid - state quantum information. At present time spintronics refer to spin electronics, the phenomena of spin - polarized transport in metals and semiconductors. The study of spin phenomena in solids characterize electrical (field - effect transistor), optical and magnetic properties of solids due to the presence of equilibrium and nonequilibrium spin populations, as well as spin dynamics. These fundamental aspects of spintronics give us as important insights about the nature of spin interaction, hyperfine, or spin exchange couplings - in solids.

Among the prime premises of quantum physics is the principle of indistinguishability of elementary particles. All electrons are by definition 'the same' and hence, after two (or more) electrons interact, it is possible to say 'who is who'. The same applies to other elementary particles and even identical atoms (e.g., two ${ }^{70} \mathrm{Ge}$ atoms are indistinguishable). Not so for isotopes. Due to their mass difference, various isotopes of the same chemical element (e.g., ${ }^{70} \mathrm{Ge}$ and ${ }^{76} \mathrm{Ge}$ ) are classical distinguishable particles. Within the framework of statistical thermodynamics, this classical distinguishability of isotopes renders isotopic mixtures a prime illustrative tool for a discussion of the Gibbs paradox. The latter refers to the (alleged) discontinuity of entropy upon the mixture of two slightly different species.

The discovery of the neutron by Chadwick in 1932 may be viewed as the birth of the strong interaction: it indicated that nuclei consists of protons and neutrons and hence the presence of a force that holds them together, strong enough to counteract the electromagnetic repulsion. It is well - known that most of the mass of an atom is concentrated in the tiny nucleus surrounded by a cloud of electrons. The nucleus in turn is formed of protons and neutrons, but these are not elementary either (see, e.g. Fig. 1 in Chapter 2 of [1]). They are actually composed of even smaller particles, called quarks. Gradually over the last century physicists built up what we now call the Standard Model (SM) of particle physics [2], which describes the particles we now believe to be fundamental and their interaction. The SM includes Quantum Chromodynamics (QCD), the theory of the strong nuclear interactions. According to this model, all matter is built from a small number of fundamental spin $1 / 2$ particles, or fermions; six quarks and six leptons. The leptons carry integral electric charge. The electron e with unit negative charge is familiar to everyone, and the charged leptons are the muon $\mu$ and tauon $\tau$. These are heavy versions of the electron. The neutral leptons are called neutrinos, denoted by the generic symbol $v$. A different 'flavor' of neutrino is paired with each 'flavor' of charged lepton, an indicated by the subscript. For example, in nuclear $\beta$ - decay, an electron e is emitted together with an electron - type neutrino, $v$. The charged muon and tauon are both unstable, and decay spontaneously to electrons, neutrinos and other particles (see, e.g. [3]). The mean lifetime of the muon is $2.2 \times 10^{-6} \mathrm{~s}$, that of the tauon only $2.9 \times 10^{-13} \mathrm{~s}$. In the theory of strong interquark forces, QCD, there are six types of strong charge, called color charges; color is just a name for an internal degree of freedom. A quark can carry one of three primary colors (say red, blue and green), and its antiquark then carries the corresponding anticolor. The quark combinations called hadrons have no color. A baryon (proton or neutron) of one red, one blue and one green quark, the combination being white (i.e. colorless) Similarly, a meson consisting of a quark of a particular color and antiquark of that anticolor, is also colorless. Gluons, unlike photons, carry a color charge, consisting of one color and one anticolor.

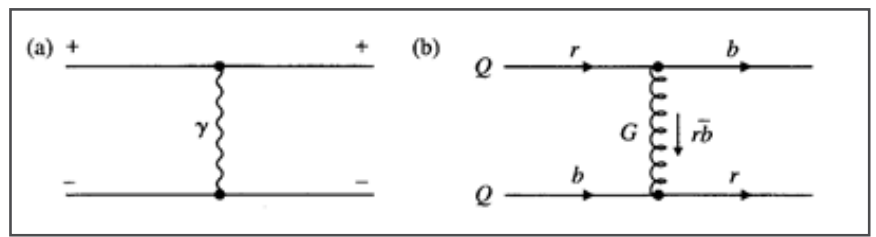

Fig. 1: Comparison of the Feynman diagrams of electromagnetic (a) and strong (b) interactions. The electromagnetic interaction in (a) involves two types of electric charge, $(+)$ and $(-)$ is mediated by an uncharged photon. In (b) the strong interquark force involves six types of color charge. The diagram depicts the interaction of a red quark with a blue quark via the exchange of a red antiblue gluon.

As an example, Fig. 1b shows a red quark $r$ interacting with a blue quark $b$ via exchange of rb gluon. note that the gluon carrier itself is endowed with a color charge (rb in our case). On this account, gluons have self - interaction, with triple (and quadrupole) gluon vertices [4]. This marks a crucial difference from Quantum Electrodynamics (QED), where the photon uncharged and there is no first - order photon - photon interaction [4]. In the jargon, QED is said to be an Abelian, and QCD is a non - Abelian theory [5]. Since the gluons carry one color and one anticolor, there should be $3^{2}=9$ possible color combinations. However, one of these turns out to be a colorless singlet (in the form $r \bar{r}+b \bar{b}+g \bar{g}[3]$ ), leaving eight active gluon states: so - called eight - fold way [6].

The diagram of Fig. 1 is drawn for the case of single -- gluon exchange, but taking into account that strong constant coupling $\alpha_{\mathrm{s}} \sim 1$, multiple exchanges, involving terms $\alpha_{\mathrm{s}}^{2}, \alpha_{\mathrm{s}}^{3}, \ldots$ must also be very probable. However, it turns out that for violent 'close' collisions at very high $\mathrm{q}^{2}, \alpha_{\mathrm{s}}<<1$ and single - gluon exchange is a good approximation, while at low $\mathrm{q}^{2}$ or, equivalently, larger distances, the coupling becomes very large and the theory is incalculable. This large - distance behavior is presumably linked with of confinement of quarks and gluons inside hadrons. Since gluons are massles, one might expect the static QCD potential to have a similar 1/r, form to that in QED. In fact the quark - antiquark potential is often taken to be of the form

$$
\mathrm{V}_{\mathrm{s}}=-(4 / 3)\left(\left(\alpha_{\mathrm{s}}\right) / \mathrm{r}\right)+\mathrm{kr}
$$

Where the first term, dominating at small $r$, arises from single - gluon exchange. It is similar to the Coulomb potential between two unit charges of

$$
\mathbf{V}_{\mathrm{em}}=-(\boldsymbol{\alpha} / \mathbf{r})
$$

The factor $4 / 3$ in (1) is plausible in view of the fact that there eight color gluon states, to be averaged over three quark colors, 
giving factor $8 / 3$ as compared with QED (one 'color' of photon and one fermion charge). This has to divide by 2 because, for historical reasons [4], a factor 2 enters into definition of $\alpha_{s}$ in terms of the square of the strong color charge. However, at larger distances the second term in (1) is dominant and is responsible for quark confinement. The value of $\mathrm{k}$ is about 0.85 $\mathrm{GeVfm}^{-1}$. In equation (1) the attractive force at large $\mathrm{r}$ is $\mathrm{dV} / \mathrm{dr}$ $=\mathrm{k}=0.85 \mathrm{Gevfm}^{-1}$ [4]. The estimation of the $\mathrm{k}$ equals $\mathrm{k}=13.9$ tonnes weight - a great deal for those tiny quarks, each weighing less than $10^{-24} \mathrm{gm}$. Because gluons carry a color charge (unlike photons which are uncharged) there is a strong gluon - gluon interaction. If one tries to pull apart the two quarks, the energy required to do so grows linearly with the string (distance) as in (1), and eventually it requires less energy to produce another quark - antiquark pair, thus involving two short string instead one long one. Thus even the most violent efforts to separate quarks just result in production of lot of quark - antiquark pairs (mesons) [3]. Since the protons and neutrons which make up the nucleus are themselves considered to be up of quarks are considered to be held together by the color force [3], the strong force between nucleons may be considered to be residual color force (see e.g. [1]).

In modern physics, particles are classified into two groups based on their properties. There are known as fermions and bosons. In the SM fermions are the fundamental particles of matter. Elementary fermions are leptons (see Table 1).

Table 1. Quarks and leptons

\begin{tabular}{|l|l|l|l|l|}
\hline \multirow{2}{*}{} & \multicolumn{3}{|l|}{ Family } & \multirow{2}{*}{ Electric charge (e) } \\
\cline { 2 - 5 } & $\mathrm{l}$ & 2 & 3 & \\
\hline \multirow{3}{*}{ Leptons } & $\overline{\mathrm{e}}$ & $\mu$ & $\tau$ & -1 \\
\cline { 2 - 5 } & $v_{\mathrm{e}}$ & $v_{\mu}$ & $v_{\tau}$ & 0 \\
\hline \multirow{2}{*}{ Quarks } & $\mathrm{u}$ & $\mathrm{c}$ & $\mathrm{t}$ & $2 / 3$ \\
\cline { 2 - 4 } & $\mathrm{d}$ & $\mathrm{s}$ & $\mathrm{b}$ & $-1 / 3$ \\
\hline
\end{tabular}

Fermions are the half - integer spin particles and described by the Fermi - Dirac statistics. The quarks and leptons are building block of matter and antimatter [1 - 3]. In the SM, the base exchange is the gluon which mediates the forces between quarks. Bosons are identical particles having zero of integer spin. Behaviors of bosons are described by the Bose - Einstein statistics. The SM [2 - 4] only consists of five elementary bosons and twelve of leptons and quarks. Besides that there are gluons and Higgs boson (Fig. 2).

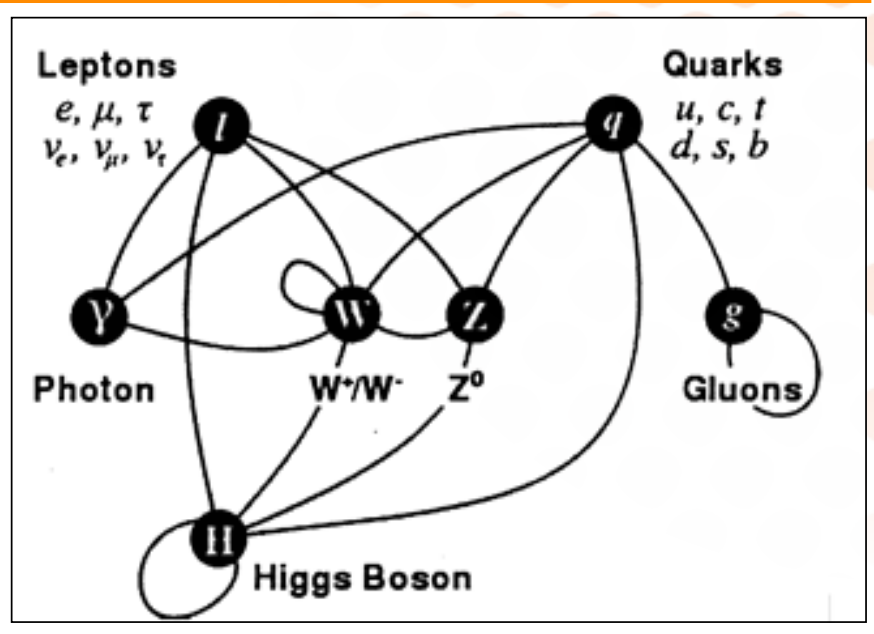

Fig. 2. Summary of interactions between particles described by the Standard Model.

\section{Experimental}

Below we briefly describe the results of the low - temperature optical spectroscopy of isotope - mixed solids. The apparatus used in our experiments has been described in several previous publication [7 - 9]. For clarity, we should mentioned here that immersion home - made helium cryostat and two identical double - prism monochromators were used. One monochromator was used for the excitation and the other, which was placed at right - angle to the first for analyzing the luminescence and scattering of light. In our experiments we investigated two kinds of crystals ( $\mathrm{LiH}$ and $\mathrm{LiD}$ ) which are different by a term of one neutron. Lithium hydride and lithium deuteride are ionic insulating crystals with simple electronic structure, four electrons per unit cell, both fairly well -described structurally (neutron diffraction) and dynamically (second -- order Raman spectroscopy) and through ab initio electronic structure simulation. Among other arguments, $\mathrm{LiH}$ and LiD are very interesting systems due to their extremely simple electronic and energy structure and to the large isotopic effects when the hydrogen ions are replaced by the deuterium ones. On the other hand, the light mass of the ions, especially $\mathrm{H}$ and $\mathrm{D}$, makes that these solids have to be considered like quantum crystals, and consequently, described theoretically by quantum theory. In a solid one deals with a large number of interacting particles, and consequently the problem of calculating the electronic wave functions and energy levels is extremely complicated. It is necessary to introduce a number of simplifying assumptions. In the first place we shall assume that nuclei in the crystalline solid are at rest. In an actual crystal this is of course never the case, but the influence of nuclear motion on the behavior of electrons may be treated as a perturbation for the case in which they are assumed to be at rest. Even with above assumption, however, we are still with a many -- electron problem which can be solved only be approximate methods. In the case of solids, the most important approximate method which has been applied extensively is the so -- called one -electron approximation. In this approximation the total wave function for the system is given by a combination of wave functions, each of which involves the coordinates of only one electron. In other words, the field seen by a given electron is 
assumed to be that of the fixed nuclei plus some average field produced by the charge distribution of all other electrons [11].

The difference between a good conductor and a good insulator is striking. The electrical resistivity of a pure metal may be as low as $10^{-10} \mathrm{ohm}-\mathrm{cm}$ at a temperature of $1 \mathrm{~K}$, apart from the possibility of superconductivity. The resistivity of good insulator may as high as $10^{22} \mathrm{ohm}-\mathrm{cm}$. The understand the difference between insulators and conductors, we shall use the band -- gap picture (Fig. 3 below). The possibility of band gap is the most important property of solids.

The single crystals of $\mathrm{LiH}$ and $\mathrm{LiD}$ were grown from the melt by the modified method of Bridgeman - Stockbarger (see [7, 8] and references quoted therein). The crystals were synthesized from ${ }^{7} \mathrm{Li}$ metal and hydrogen of $99.7 \%$ purity and deuterium of $99.5 \%$ purity. Virgin crystals had a slightly blue - grey color, which can be attributed to nonstoichiometric excess of lithium present during the grown cycle.

On annealing for several days (up to 20 ) at $500^{\circ} \mathrm{C}$ under $\sim 3$ atm of hydrogen or deuterium, this color could be almost completely eliminated. Because of the high reactivity and high hygroscope of investigated crystals an efficient protection against the atmosphere was necessing. Taking into account this circumstance, we have developed special equipment which is allowed to prepare samples with a clean surface cleaving there in the bath of helium cryostat with normal or superfluid liquid helium [12]. The samples with such surface allow to perform measurements during 15 hours. An improved of $\mathrm{LiH}$ (LiD) as well as mixed crystals were used in the present study. In spite of the identical structure of all free - exciton luminescence spectra, it is necessary to note a rather big variation of the luminescence intensity of the crystals from the different batches observed in experiment.

We should remind very briefly about the electronic excitations in solids. According to modern concept, the excitons can be considered [10] as the excited of the N - particles system: An electron from the valence band (see Fig. 3) is excited into the conduction band.

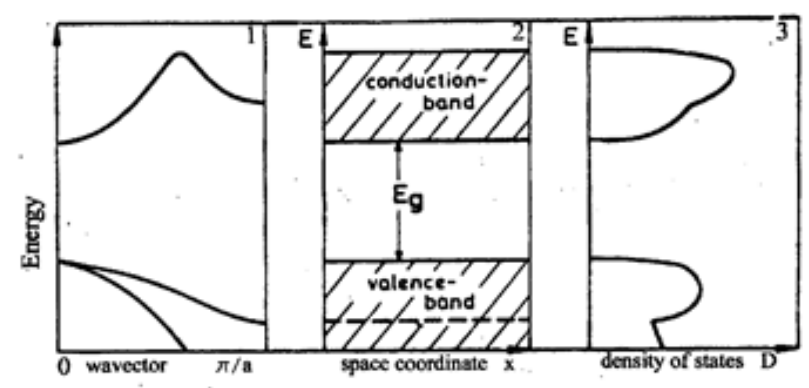

Fig. 3: Various possibilities to present the band - structure of homogeneous, undoped insulator (semiconductor). 1 the dispersion relation, i.e. the energy $E$ as a function of the wave vector $k, 2$ - the energy regions of allowed and forbidden states as function of a space coordinate $x$ and, 3 - the density of states (all curves are schematic ones).

The attractive Coulomb potential between the missing electron in the valence band, which can be regarded as a positively charged hole, and the electron in the conduction band gives a hydrogen - like spectrum with an infinitive number of bound state and ionization continuum (Fig. 4).

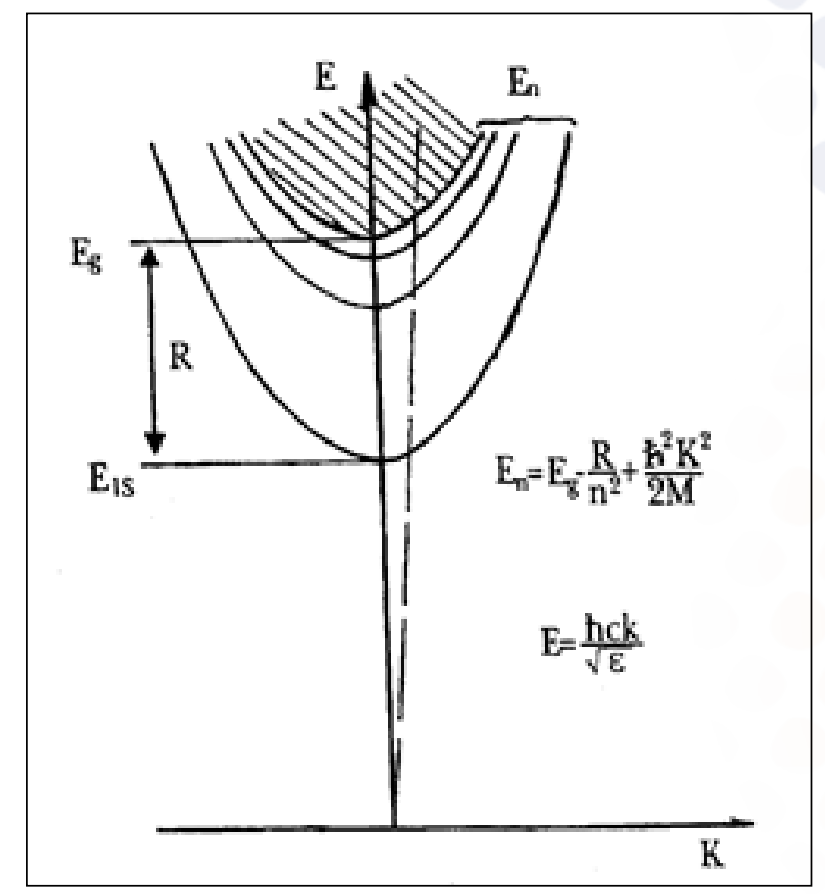

Fig. 4: Discrete and continuous (hatched area) Wannier - Mott exciton energy spectrum taking into account its kinetic energy. The broken line connects to the dispersion of light in the medium. $R$ - is the exciton binding energy, $n$ $=1,2,3, \ldots .$.

In our short review we call the bound states of electron - hole $(\mathrm{e}-\mathrm{h})$ pairs exciton states (exc), while we refer to ionized e $\mathrm{h}$ pairs as free carriers. However, the expression free carriers does not imply that the effect of the strong Coulomb forces between electronic excitation could be neglected. Thus, an exc - state can be built by appropriate superposition of $\mathrm{e}-\mathrm{h}$ pairs, which in a simple two - band model for cubic crystal symmetry is given (more details see [10]). In our experiments we have investigated the low - temperature optical spectra (reflection, photoluminescence and scattering of light) of $\mathrm{LiH}_{\mathrm{x}} \mathrm{D}_{1-\mathrm{x}}$ crystals $(0 \leq \mathrm{x} \leq 1)$ which are different by term of one neutron from each other.

\section{Results}

The usual way to determine the optical properties of a solid is to shine monochromatic light onto an appropriate sample and then to measure the reflectance or transmittance of the sample as a function of photon energy. In the spectral region of greatest interest is generally quite high, so that often a negligible small fraction of the incident light is transmitted. It is the main reason, that the most experiments are measurements of the reflectivity. The mirror reflection spectra of mixed and pure LiH and LiD crystals, cleaved in superfluid helium are presented on Fig. 5. All spectra have been measured with the same apparatus under the same conditions. As can clearly see in Fig. 5 all spectra exhibit a similar long -- wave structure. 
As the deuterium concentration increases, the long -- wave maximum ( $\mathrm{n}=1 \mathrm{~S}$ excitons [11]) broadens and shifts towards the shorter wavelengths. The energy values of exciton maxima for pure and mixed crystals at $2 \mathrm{~K}$ are presented in Table 2 .

\begin{tabular}{|l|l|l|l|l|l|}
\hline & $\mathrm{LiH}$ & $\mathrm{LiH}_{0.82} \mathrm{D}_{0.18}$ & $\mathrm{LiH}_{0.40} \mathrm{D}_{0.60}$ & $\mathrm{LiD}$ & $\mathrm{Li}^{6} \mathrm{H}(78 \mathrm{~K})$ \\
\hline $\mathrm{E}_{1}$ & 4950 & 4967 & 5003 & 5043 & 4939 \\
\hline $\mathrm{E}_{2}$ & 4982 & 5001 & 5039 & 5082 & 4970 \\
\hline $\mathrm{E}_{\mathrm{b}}$ & 42 & 45 & 48 & 52 & 41 \\
\hline $\mathrm{E}_{\mathrm{g}}$ & 4992 & 5012 & 5051 & 5095 & 4980 \\
\hline
\end{tabular}

Table 2. Values of the energy of maxima (in $\mathrm{meV}$ ) in exciton reflection spectra of pure and mixed crystals at $2 \mathrm{~K}$, and energies of exciton binding energy $E_{b}$, band - to - band transitions $\mathbf{E}_{\mathrm{g}}$.

As demonstrated early (see, e.g. book [11) most low - energy electron excitation in $\mathrm{LiH}$ crystals are the large - radius excitons [10].

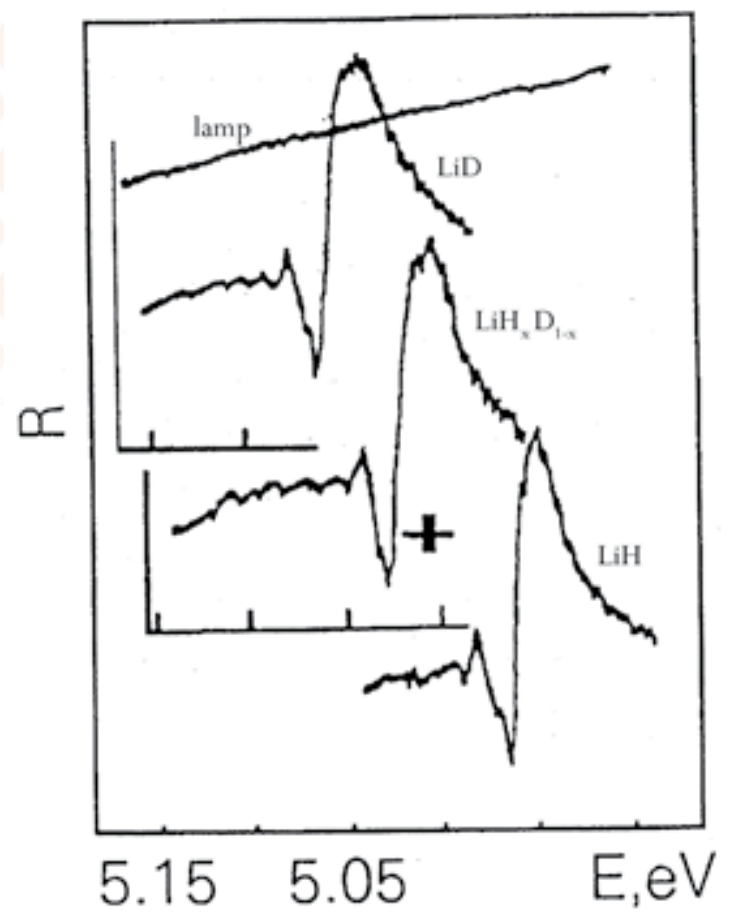

Fig. 5: Mirror reflection spectra in indicated crystals at $2 \mathrm{~K}$ cleaved in superfluid helium. Light source without crystals, curve is lamp.

Going back to Fig. 5, it is hard to miss the growth of $\Delta_{12}$, which in hydrogen - like model [10] causes an increase in exciton Rydberg $\left(\mathrm{E}_{\mathrm{b}}\right)$ with the replacement of isotopes (for details see [11]). The single - mode nature of exciton reflection spectra of mixed crystals $\mathrm{LiH}_{\mathrm{x}} \mathrm{D}_{1-\mathrm{x}}$ agrees qualitatively with the results obtained with the virtual crystal model [13]. All of these results are documented in Table 2, where the variation of $\mathrm{E}_{\mathrm{g}}, \mathrm{E}_{\mathrm{b}}$ are shown at the isotope effect. We should highlight here that the most prominent isotope effect is observed in $\mathrm{LiH}$ crystals, where the dependence of $\mathrm{E}_{\mathrm{g}}=\mathrm{F}\left(\mathrm{C}_{\mathrm{D}}\right)$ is also observed and investigated (see below Fig. 9 ).
Photoluminescence is the optical radiation emitted by a physical system (in excess of the thermal equilibrium blackbody radiation) resulting from excitation to a nonequilibrium state by irradiation with light. Photoluminescence is also rapidly evolving into major basic research tool comparable to absorption (reflection) measurements in importance. Two reason for this stand out as significant. First is the sensitivity of the luminescence technique. It often happens that features which are just discernible in absorption will completely dominate the luminescence spectra. The converse is also sometimes true, making luminescence and absorption (reflection) complementary techniques. Second is the simplicity of data collection. In last half- century the luminescence method has become one of the most common techniques for studying excitons in dielectrics and semiconductors. While the structure of spectra of fundamental reflection (absorption) depends on the internal degrees of freedom of Wannier -- Mott exciton, the structure and shape of the luminescence spectrum are determined primarily by its external degrees of freedom. The latter are associated with the translation motion of large -- radius exciton as a whole, with the translation mass $\mathrm{M}=\mathrm{m}_{\mathrm{e}}+$ $m_{h}$, where $m_{e}$ and $m_{h}$-- effective masses of electron and hole, respectively.

Free exciton luminescence is observed when $\mathrm{LiH}(\mathrm{LiD})$ crystals are excited in the midst of the fundamental absorption. The spectrum of free exciton photoluminescence of $\mathrm{LiH}$ crystals cleaved in superfluid helium consists of a narrow (in the best crystals, its half - width is $\Delta \mathrm{E} \leq 10 \mathrm{meV}$ ) phononless emission line and its broader phonon repetitions, which arise due to radiated annihilation of excitons with the production of one to five longitudinal optical (LO) phonons (see Fig. 6).

The phononless emission line coincides in an almost resonant way with the reflection line of the exciton ground state which is indication of the direct electron transition $X_{1}-X_{4}$ of the first Brillouin zone [14].The lines of phonon replicas form an equidistant series biased toward lower energies from the resonance emission line of excitons. The energy difference between these lines in $\mathrm{LiH}$ crystals is about $140 \mathrm{meV}$, which is very close to the calculated energy of the LO phonon in the middle of the Brillouin zone [15] and which was measured in (see, e.g. [8] and references quoted therein). The isotopic shift of the zero - phonon emission line of LiH crystals equals $103 \mathrm{meV}$. As we can see from Fig. 6 the photoluminescence spectrum of LiD crystals is largely similar to the spectrum of intrinsic luminescence of $\mathrm{LiH}$ crystals. There are, however, some distinctions one is related. Firstly the zero - phonon emission line of free excitons in LiD crystals shifts to the short - wavelength side on $103 \mathrm{meV}$. These results directly show the violation of the strong conclusion (see, e.g. $[1,16])$ that the strong force does not act on leptons. The second difference concludes in less value of the LO phonon energy, which is equal to $104 \mathrm{meV}$. The simplest approximation, in which crystals of mixed isotopic composition are treated as crystals of identical atoms having the average isotopic mass, is referred to as virtual crystal approximation (VCA) [13]. 


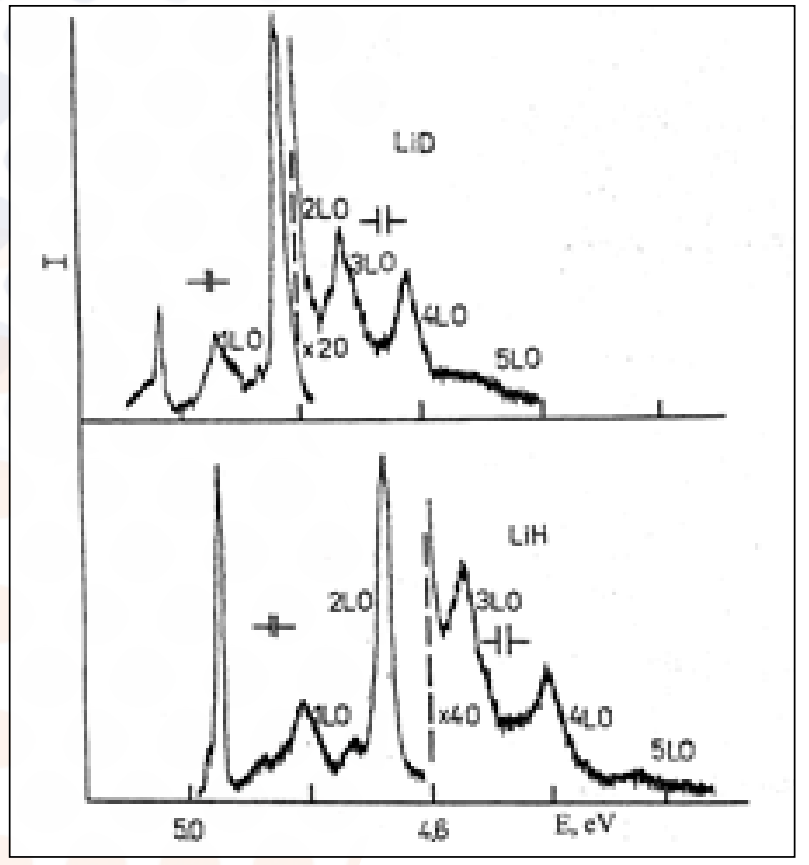

Fig. 6: Photoluminescence spectra of free excitons at $2 \mathrm{~K}$ in LiH and LiD crystals cleaved in superfluid helium.

When light is excited by photons in a region of fundamental absorption in mixed $\mathrm{LiH}_{\mathrm{x}} \mathrm{D}_{1-\mathrm{x}}$ crystals at low temperature, line luminescence is observed (Fig. 6), like in the pure $\mathrm{LiH}$ and $\mathrm{LiD}$ crystals. As before [8], the luminescence spectrum of crystals cleaved in superfluid liquid helium consists of the relatively zero - phonon line and its wide LO replicas. For the sake of convenience, and without scarfing generality, Fig. 7 shows the lines of two replicas. Usually up to five LO repetitions are observed in the luminescence spectrum as described in detail in $[8,11]$. In Fig. 7 we see immediately that the structure of all three spectra is the same. The difference is in the distance between the observed lines, as well as in the energy at which the luminescence spectrum begins, and in the half - width of the lines (see, also Table 2).

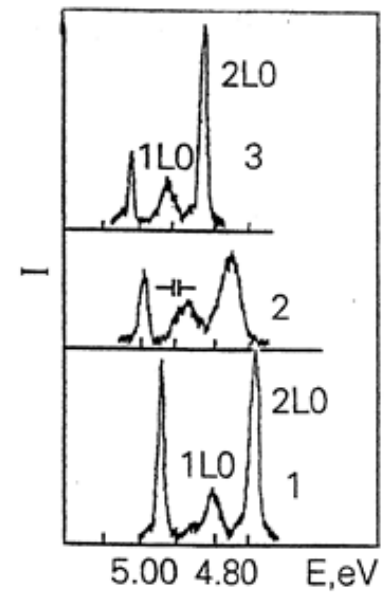

Fig. 7. Photoluminiscence spectra of free excitons in $\mathbf{~ L i H}$ (1), $\operatorname{LiH}_{x} D_{1-x}(2)$ and $\mathrm{LiD}(3)$ crystals cleaved in superfluid helium at $2 \mathrm{~K}$. Spectrometer resolution is shown.
At the excitation below the intrinsic absorption edge $\left(\mathrm{E}_{\mathrm{n}=1 \mathrm{~s}}=5.043 \mathrm{eV}\right.$ for LiD [11]) we have succeeded in observing the multiphonon resonance Raman scattering (RRS) with the creation of up four phonons (Fig. 8). Indeed, the energy difference between peaks in the RRS spectrum is equal the energy of the LO phonons in the center of the Brillouin zone $[8,15]$.

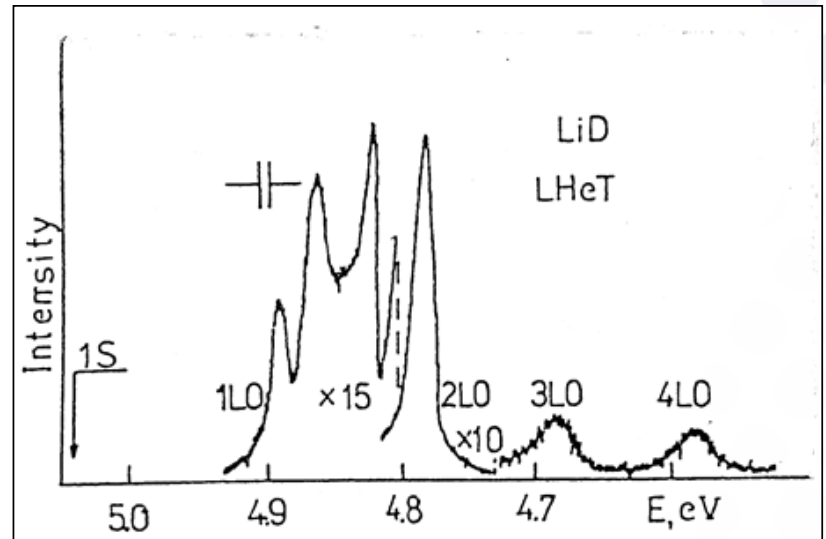

Fig. 8: Resonant Raman scattering of a LiD crystals at the excitation $E=4.992 \mathrm{eV}$ at $4.2 \mathrm{~K}$.

To pay attention the large half - width of observable lines in the RRS spectrum. As was shown in the paper [11] their half - width are always larger than of the excitation line. The proximity of the exciting light frequency to the energy of exciton transitions leads to an essential modification of the selection rules for light scattering. The presence of the second - order TO $(\Gamma)\left(\hbar \omega_{\mathrm{TO}(\Gamma)}\right.$ $=76 \mathrm{meV}$ for $\mathrm{LiH}$ ) in the RRS spectrum may be explained by a relatively strong scattering deformation mechanism in these crystals, where, however the main mechanism, as was seen from both figures, is Fröchlich mechanism of intraband scattering. The longwavelength displacement of the excitation line frequency relatively exciton resonance a monotonic decrease the intensity of RRS spectrum as whole more than 60 - fold in both LiH and LiD crystals [11].

Fig. 9: shows the concentration dependence of the energy of interband transition $\mathrm{E}_{\mathrm{g}}$ (see Fig. 2). As can be seen from Fig. 9, VCA method (the straight dashed line) cannot describe observed experimental results. This dependence has a nonlinear character. 


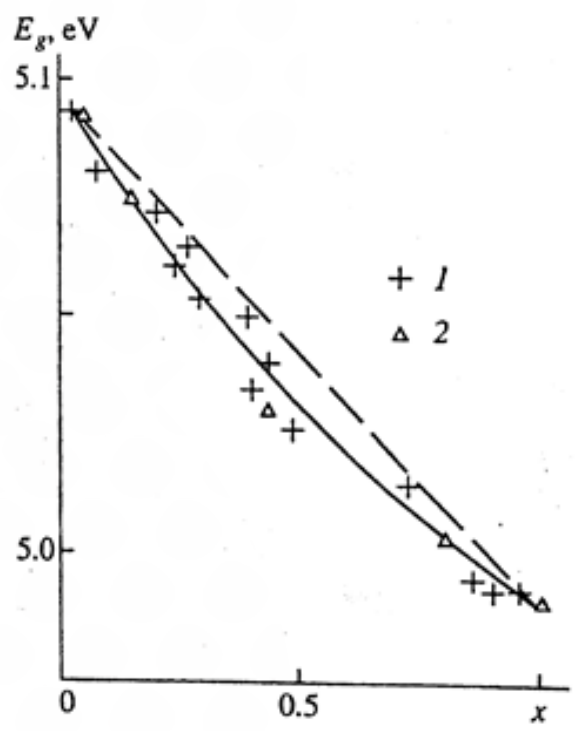

Fig. 9: Dependence of the interband transition strong interaction strength energy $\mathrm{Eg}$ in mixed crystals on the concentration $x$ number of neutrons $N$. The straight dashed line is the linear dependence of coupling constant strong interaction $\alpha_{s}=f(N)\left[E_{g}=f(x)\right]$ in the virtual model. The solid line correspods to calculation using the polynom of second degree $\alpha_{\mathrm{s}}=\alpha_{\mathrm{s}}(\mathrm{LiD})+\left[\alpha_{\mathrm{s}}(\mathrm{LiH})-\alpha_{\mathrm{s}}(\mathrm{LiD})-\mathrm{b}\right] \mathbf{x}-\mathrm{bx}^{2}$, where $b=0.046 \mathrm{eV}$ is curvature parameter [21]. Points derived from the reflection spectra indicated by crosses, and those from luminescence spectra by triangles.

Comparison the all experimental results on the luminescence (reflection) and light scattering in the crystals which differ by a term of one neutron only is allowed to the next conclusions;

1. At the adding one neutron (using LiD crystals instead $\mathrm{LiH}$ ones) is involved the increase exciton energy on $103 \mathrm{meV}$.

2. At the addition one neutron the energy of LO phonons is decreased on the $36 \mathrm{meV}$, that is direct seen from luminescence and scattering spectra.

\section{Discussion}

Traditionally nuclear - electron interaction (in our case neutron - electron interaction) taking into account the solving of Schrödinger equation using Born - Oppenheimer (adiabatic) approximation [17]. Since electrons are much faster and lighter than the nuclei by a factor nearly 2000 the electron charge can quickly rearrange itself in response to the slower motion of the nuclei, and this is the essence of the Born - Oppenheimer approximation. This approximation results the omission of certain small terms which result from the transformation. As was shown in [18] the eigenvalue (energy of the electronic Schrödinger equation) (equation 6 in [18]) depends on the nuclear charges through the Coulomb potential, but it doesn't include any references to nuclear mass and it is the same for the different isotopes. The independent of the potential energy (the eigenvalue of the Schrödinger equation) is the essence adiabatic approximation. However, we must repeat, that the
Born - Oppenheimer approximation is the standard anzatz to the description of the interaction between electrons and nuclei in solids (see, e.g. [19]). The last result is forcing us to search for new models and mechanisms of nuclear - electron interaction including results of subatomic physics, e.g. hadron - lepton interaction [3].

Out of four known interactions, three are described by SM - the electromagnetic, weak and strong ones. The first two of them have a common electroweak gauge interaction behind them. The symmetry of this interaction $\mathrm{SU}(2)_{\mathrm{L}} \mathrm{X} \mathrm{U}(1)_{\mathrm{Y}}$ manifests itself at energies higher than $\sim 200 \mathrm{GeV}$. At lower energies, this symmetry is broken down to $\mathrm{U}(1)_{\mathrm{EM}} \neq \mathrm{U}(1)_{\mathrm{Y}}$ (the electroweak symmetry breaking): in SM this breaking is related to the vacuum expectation value of a scalar field [3]. The strong interaction in SM is described by the QCD, a theory with the gauge group $\mathrm{SU}(3)_{\mathrm{C}}$. The effective coupling constant of this theory grows when the energy decreased. As a result, particles which feel this interaction cannot exist as free states and appear only in the form of bound states called hadrons (see, also [1]). Most of modern methods of quantum field theory work at small values of coupling constant, $\alpha_{s},[20]$, that is, for QCD, at high energies. Quarks and leptons, the so - called SM matter fields, are described by fermionic fields. Quarks take part in strong interactions and compose observable bound state hadrons. Both quarks and leptons participate in the electroweak interaction. The matter fields constitute three generation: particles from different generation (see Table 1) interact identically but have different masses (see, e.g. [16]). For the case of neutrino, Yukawa interactions are forbidden as well, so neutrinos are strictly massless in SM (see, however $[1,16]$ and references therein)). The gauge bosons, which are carriers of interactions, are massless for unbroken gauge groups $\mathrm{U}(1)$ $\mathrm{EM}$ (electromagnetism - photons) and $\mathrm{SU}(3)_{\mathrm{C}}(\mathrm{QCD}$ - gluons), masses of $\mathrm{W}^{ \pm}$and $\mathrm{Z}^{\circ}$ bosons are determined by the mechanism of electroweak symmetry breaking. It should be noted that the forces between the quarks must be long rang, because the gluons have zero mass. This does not imply that forces between hadrons are also long range, because hadrons have zero color charges overall. The forces between the colorless hadrons are the residues of the forces between their quark constituents, and cancel when the hadrons are far apart.

Returning to our non - accelerator experimental results, we should underline that in this paper we measure the strong nuclear interaction in crystals which differ by term of one neutron from each other. When we add one neutron in the hydrogen nucleus, we artificial activated of the strong interaction in deuterium nucleus. As far as the gravitation, electromagnetic and weak interactions are the same in both kind crystals $(\mathrm{LiH}$ and LiD), it only emerges the strong interaction in deuterium nucleus. Therefore a logical conclusion is made that the renormalization of the energy of electromagnetic excitations (isotopic shift equals $0.103 \mathrm{eV}$ ) is carried out by strong nuclear interaction. The short range character of the strong interaction of nucleons does not possess direct mechanism of the elementary excitation (electrons, excitons, phonons) energy renormalization, which was observed in our low temperature 
experiments. Second reason that the interpretation of our experimental results is very difficult task because they are first demonstration of the violation of the strong conclusion in nuclear and particles physics that the strong nuclear force does not act on the colorless leptons (see, e.g. [1, 2]). Moreover we have some contradictions taking into account that the forces between quarks must be long range, because the gluons have zero mass. But as was mentioned above, the short range when forces between the colorless hadrons are the residues of the forces between their quark constituents, and cancel when the distance between hadrons is more than nuclear size [3]. We can see that the nuclear size transforms long range interaction in the short range strong one. It is very old question which up to present time has not any theoretical explanation.

In spite of above discussion, at present time we can distinguish the following mechanisms of the isotopic shift zero phonon line:

1. Long range electric field of the neutron's quarks. This mechanism owing to the confinement quarks is limited by the boundary of the neutron.

2. The possible new structure of the quarks and leptons so - called preons (see e.g. $[1,21]$ and references quoted therein).

3. The most likely mechanism of the neutron - lepton interaction is connected to the magnetic - like strong field of neutron 's quarks [22]. Taking into account anomalous magnetic moment of the neutron $[22,23]$ in the paper [24] was obtained the value of strong coupling constant $\alpha_{s}=2.4680$. Quite large value in comparison with the accelerator technique value $\alpha_{\mathrm{s}}\left(\mathrm{M}_{\mathrm{z}}\right)=0.1198$ [20]. The large value $\mathrm{s}$ is thus justified to think that residual strong forces acting beyond nucleon could exist. A possible interpretation is to assume that in addition to the 8 gluons [6] predicted by QCD SU(3) group (see above) there is a ninth gluon color singlet [3]

$$
\mathrm{g}_{9}=(1 /(\sqrt{ } 3))(\mathrm{r} \overline{\mathrm{r}}+\mathrm{g} \overline{\mathrm{g}}+\mathrm{b} \overline{\mathrm{b}})
$$

This massless photon - like gluon may be strongly interacts between nucleons (neutrons) and leptons (electrons) (see, also [2 - 4]). Returning to Fig. 9 we can note that our measurements permit to obtain value of strong coupling constant from $\alpha_{\mathrm{s}}=2.4680$ (pure LiD crystals) to $\alpha_{\mathrm{s}}=0$ (pure LiH crystals). Moreover, in Fig. 10 we show the dependence of as on neutron's number in different substances. We can see as early in the case of pure LiD crystals we have non - linear dependence of as on the neutron's number in different substances, which as in the case $\mathrm{LiH}_{x} \mathrm{D}_{1-\mathrm{x}}$ doesn't have any theoretical explanation yet.

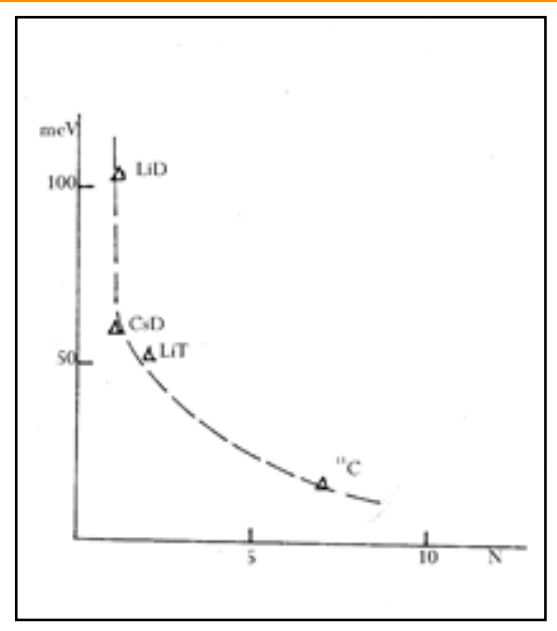

\section{Fig. 10: The dependence of the strong force on the number of neutrons in different substances.}

Thus, the above in many tentative interpretation of describing non - accelerator experimental results does not find consisted explanation at the change of strong interaction leaving to another mystery of SM $[2,4]$. We should remind that intrinsic contradiction of Standard Model is already well - known. Really, the Lagrangian of quantum chromodynamics (theory of the strong interaction) has the next form (see, e.g. [12]):

$$
\mathrm{L}=\mathrm{i} \sum_{\mathrm{q}} \bar{\Psi}_{\mathrm{q}}^{\mathrm{a}}\left(\nabla_{\mu} \gamma_{\mu}+\mathrm{im} \mathrm{m}_{\mathrm{q}}\right) \Psi_{\mathrm{q}}^{\mathrm{a}}-\frac{1}{4} \mathrm{G}_{\mu v}^{\mathrm{n}} \mathrm{G}_{\mu v}^{\mathrm{n}},
$$

\section{where}

$\nabla_{\mu}=\partial_{\mu}-$ ig $\frac{\lambda^{n}}{2} A_{\mu}^{n}$,

$\mathrm{G}_{\mu v}^{\mathrm{n}}=\partial_{\mu} \mathrm{A}_{\mu}^{\mathrm{n}}-\partial_{\mathrm{n}} \mathrm{A}_{\mu}^{\mathrm{n}}+\mathrm{gf}^{\mathrm{nm}} \mathrm{A}_{\mu}^{\mathrm{n}} \mathrm{A}_{v}^{l}$.

$\Psi_{\mathrm{a}}{ }^{\mathrm{a}}$ and $\mathrm{A}_{\mu}{ }^{\mathrm{n}}$ are quark and gluon fields, $\mathrm{a}=1,2,3, \ldots 8$ are color indices, $\lambda^{n}$ and flum are Gell - Mann matrices and f symbols, $\mathrm{mq}$ - are bare (current) masses, $\mathrm{q}=\mathrm{u}, \mathrm{d}, \mathrm{s}, \mathrm{c}, \ldots$ different quarks. It is common place $[4,25]$ that the Lagrangian (4) contains the members which describe both free motion and interaction between quarks and gluons, which is defined by the strength couple g. Spacing of which it is necessary to remark that although the Lagrangian (4) possesses rather attractive peculiarities (see, also $[1-4,16,20,26]$ ), its eigenstates are the quarks and the gluons which are not observed in free states $[4,26,27]$. The observed hadrons in the experiment don't eigenstates in QCD. It is obvious to expect that the modern theory of QCD should finally overcome these difficulties, for example inserting QCD in QED.

\section{Conclusion}

The artificial activation of the strong nuclear interaction by adding one (two or more) neutrons in atomic nuclei leads it to the direct observation of the strong interaction in low - temperature optical spectra of solids. This conclusion opens new avenue in the investigation of the constant of strong nuclear interaction in the wide value range by means the condensed matter alike traditional methods. Our experimental nonaccelerator results may shed light on a number of fundamental puzzles in modern 
physics, particularly on the unification of forces. Experimental observation of the renormalization of the elementary excitation energy of solids by the strong nuclear interaction stimulates its count in the process of description of the elementary excitations dynamics in quantum electrodynamics. Besides, we should highlight that such important information has been obtained via rather simple and inexpensive experimental physics equipment. Present article continuous to develop between nuclear, high energy and condensed matter physics. Our brief review continues to develop the connection between nuclear and condensed matter physics.

\section{Acknowledgements}

Many thanks to Dr. G. Tymar for reading my manuscript, K. Walker and G.A. Plehhanov for improving my English.

\section{References}

1. V.G. Plekhanov, Modern View of the Origin of Isotope Effect (LAP, LAMBERT, Academic Publishing, Saarbrücken, Germany, 2018).

2. W.N. Cottingham, D.A. Greenwood, An Introduction to the Standard Model of Particle Physics (CUP, Cambridge, 2007).

3. G. Griffiths, Introduction to Elementary Particles (Wiley WC, Weinheim, 2008).

4. J.F. Donoghue, E.Golowich, B.R. Holstein, Dynamics of the Standard Model (CUP, Cambridge, 2008).

5. See, e.g. G. Arfken, Mathematical Methods for Physicists (Academic Press, New York and London, 1968).

6. M. Gell - Mann, Y. Neeman. The Eightfold Way (Benjamin, Reading,Mass., 1964).

7. V.G. Plekhanov, Experimental manifestation of the effect of disorder on exciton binding energy in mixed crystals, Phys. Rev. B53, 9558 - 9560 (1996).

8. V.G. Plekhanov, Isotope effects in lattice dynamics, Phys. - Uspekhi (Moscow) 46, 553 - 579 (2003).

9. V.G. Plekhanov, Renormalization the energy of elementary excitations in solids by the strong nuclear interaction, Universal J. Phys. and Appl. 11, 6 - 12 (2017).

10. R.S. Knox, Theory of Excitons (Academic Press, New York - London, 1963).

11. V.G. Plekhanov, Giant Isotope Effects in Solids (Stefan University Press, La Jola, CA, 2004).

12. V.G. Plekhanov, Macroscopic manifestation of the strong nuclear interaction in the optical spectra of solids, in, Proc. ISINN - 25 (Dubna, Russia, 2018) 49 - 56.

13. I. Nordheim, Zur Elektrontheorie der metalle, Ann. Phys. (Leipzig) 401, 641 - 678 (1931).

14. S. Baroni, G. Pastori Parravichini, G. Pezzica, Quasiparticle band structure of lithium hydride, Phys. Rev. B32, 4077 4083 (1985).

15. J.L. Verble, J.L Warren and J.L. Yarnell, Lattice dynamics of lithium hydride, ibid, 168, 980 - 989 (1968).

16. E.M. Henley, A. Garcia, Subatomic Physics (World Scientific Publishing Co., Singapore, 2007).

17. M. Born and J.R. Oppenheimer, On the quantum theory of molecules, in, Quantum Chemistry (Classic Scientific Paper) (World Scientific Publishing Co., Singapore, 2000).

18. V.G. Plekhanov, Measurements of the wide value range of strong interaction coupling constant, SSRG - IJAP, 6, 32 - 37 (2019).

19. R.M. Martin, Electronic Structure - Basic Theory and Practical Methods (CUP, Cambridge, 2004).

20. A. Deur, S.J. Brodsky, G.F. Teramond, The QCD running coupling, Prog. Part. Nucl. Phys. 90, 1 - 74 (2016).

21. V.G. Plekhanov, A possible signature of neutron quarks - leptons via gluon interaction in solids, in, Proc. 21 Int. Conf. on Condensed Matter Nuclear Science, Colorado State University, Fort Collins, USA, June 2018, 503 - 511.

22. L.L. Foldy, Neutron - electron interaction, Rev. Mod. Phys. 30, 471 - 481 (1958).

23. V.G. Plekhanov, Necessity additions, Phys. - Uspekhi (Moscow) 61, 449 - 450 (2019).

24. V.G. Plekhanov and J.G. Buitrago, Evidence of residual strong interaction at nuclear - atomic level via isotopic shift in LiH - LiD crystals, Prog. Phys. 15, 68 - 71 (2019).

25. B.L. Ioffe, The origin mass and experiments on future high - energy accelerators experiments, Phys. - Uspekhi (Moscow) 49, 1077 - 1078 (2006).

26. A.S. Kronfeld, Lattice gauge theory and the origin of mass, in, One Hundred Years of Subatomic Physics, ed. by E. Henley and S. Ellis (World Scientidc Publication, Singapore, 2013).

27. V.G. Plekhanov, Phenomenology of the origin of isotope effect, PSIJ 18, 1 - 11 (2019).

Copyright: (C)2020 V.G. Plekhanov. This is an open-access article distributed under the terms of the Creative Commons Attribution License, which permits unrestricted use, distribution, and reproduction in any medium, provided the original author and source are credited. 